\title{
GRAPHS WITH A LARGE CAPACITY
}

\section{ROSENFELD}

Abstract. A constructive method for obtaining graphs with a relatively large capacity is given. The method uses products of graphs.

Introduction. In this note we present a constructive method for obtaining graphs with a relatively large capacity and obtain an upper bound for the capacity $\theta(G)$ of a graph $G$. The capacity of a graph was introduced by Shannon [5], for investigations of problems concerning noisy channels in information theory. If $\mu(G)$ is the maximal number of independent vertices in the graph $G$, it is well known [5], that $\theta(G) \geqq \mu(G)$. Our method will yield for every $k \geqq 0$, a graph $G_{k}$ such that $\theta\left(G_{k}\right) \geqq k \cdot \mu\left(G_{k}\right)$. The construction is based on the composition of graphs introduced by Harary [1].

The definitions and generally accepted notations throughout this paper will be those used in Harary [2]. $\mu(G)$ will denote the maximal number of independent vertices in $G$. Since the strong product and composition of graphs (for definitions see Harary [1]) are associative, powers of $G$ with respect to each one of them is well defined. We denote those powers by $G^{n}$ and $G^{[n]}$ resp. The capacity of $G$ is defined by

$$
\theta(G)=\sup _{n} \mu\left(G^{n}\right)^{1 / n}
$$

Upper bound for $\theta(G)$ : If $A$ and $B$ are independent sets in $G$ and $H$ resp., $A \times B$ is independent in $G \times H$, hence

$$
\mu(G \times H) \geqq \mu(G) \cdot \mu(H) \Rightarrow \mu\left(G^{n}\right) \geqq \mu^{n}(G) \Rightarrow \theta(G) \geqq \mu(G) .
$$

To obtain an upper bound, we consider the function $\alpha(G)$ introduced in [4], and defined as follows: Let $V(G)=\left\{g_{1}, \cdots, g_{n}\right\} \cdot\left\{C_{1}, \cdots, C_{s}\right\}$ is a fixed ordering of all maximal complete subgraphs of $G . \alpha_{i}^{j}=1$ if $g_{i} \in C_{j}, \alpha_{i}^{j}=0$ otherwise

$$
\begin{aligned}
& P(G)=\left\{\left(x_{1}, \cdots, x_{n}\right) \mid x_{i} \geqq 0, \sum_{i=1}^{n} \alpha_{i}^{j} x_{i} \leqq 1,1 \leqq j \leqq s\right\}, \\
& \alpha(G)=\max _{x \in P(G)} \sum_{i=1}^{n} x_{i}, \quad x=\left(x_{1}, \cdots, x_{n}\right) .
\end{aligned}
$$

Received by the editors December 5, 1969.

AMS subject classifications. Primary 0540; Secondary 9420.

Key words and phrases. Graphs, independence number, capacity of graphs, composition, strong product. 
THEOREM 1. If $G$ is an ordinary graph then

$$
\theta(G) \leqq \alpha(G) .
$$

Proof. Let $H$ be any graph and let $A \subset V(G \times H)$ be a maximal independent set of vertices in $G \times H$ (card $A=\mu(G \times H))$. Let

$$
A_{i}=\left\{h \mid\left(g_{i}, h\right) \in A\right\}, \quad A_{i}^{\prime}=\left\{\left(g_{i}, h\right) \mid\left(g_{i}, h\right) \in A\right\} .
$$

Obviously, $\left\{A_{i}^{\prime}\right\}$ is a disjoint decomposition of $A$. Hence

$$
\sum_{i=1}^{n} \operatorname{card} A_{i}^{\prime}=\mu(G \times H) .
$$

By the definition of $A_{i}$ and the independence of $A$ we have card $A_{i}$ $=$ card $A_{i}^{\prime}, A_{i}$ is an independent set in $H$. Let

$$
x_{i}=\operatorname{card} A_{i} \cdot 1 / \mu(H) .
$$

We will show first that $\left(x_{1}, \cdots, x_{n}\right) \in P(G)$. For simplicity of notation, we may assume without loss of generality, that $C_{j}=\left\{g_{1}, \cdots, g_{k}\right\}$. Since $A$ is independent, and $\left(g_{i}, g_{j}\right) \in E(G), 1 \leqq i, j \leqq k$, we must have $A_{i} \cap A_{j}=\varnothing$ and $A_{i} \cup A_{j}$ is an independent set in $H$. By the same argument, $\mathrm{U}_{1=1}^{k} A_{i}$ is an independent set in $H$ and the union is disjoint. Hence

$$
\mu(H) \sum_{i=1}^{n} \alpha_{i}^{j} x_{i}=\sum_{i=1}^{k} \operatorname{card} A_{i}=\text { card } \bigcup_{i=1}^{k} A_{i} \leqq \mu(H) \Rightarrow \sum_{i=1}^{n} \alpha_{i}^{j} x_{i} \leqq 1 .
$$

Therefore $\left(x_{1}, \cdots, x_{n}\right) \in P(G)$. Hence we have

$$
\begin{array}{cc}
\alpha(G) \geqq \sum_{i=1}^{n} x_{i}=\frac{1}{\mu(H)} \sum_{i=1}^{n} \operatorname{card} A_{i}=\frac{1}{\mu(H)} \sum_{i=1}^{n} \operatorname{card} A_{i}{ }^{\prime}=\frac{\mu(G \times H)}{\mu(H)} \\
\Rightarrow \quad \alpha(G) \cdot \mu(H) \geqq \mu(G \times H) .
\end{array}
$$

Since $\alpha(G) \geqq \mu(G)[4]$, by induction we obtain

$$
\mu\left(G^{n}\right) \leqq \mu\left(G^{n-1}\right) \alpha(G) \leqq \alpha^{n}(G) \Rightarrow \theta(G) \leqq \alpha(G) .
$$

REMARK. In [4], it was shown that $\mu(G \times H)=\mu(G) \cdot \mu(H)$ for all graphs $H$ iff $\alpha(G)=\mu(G)$. Hence for such graphs we have $\theta(G)=\alpha(G)$ $=\mu(G)$. Since the capacity of no other graphs is known nothing else can be said about the upper bound established above.

Theorem 2. For every $k>0$, there exists a graph $G_{k}$ such that $\theta\left(G_{k}\right) \geqq k \mu\left(G_{k}\right)$. 
Proof. Let $G_{0}$ be a self-complemented graph with $n$ vertices such that $\mu\left(G_{0}\right) \cdot \mu\left(\bar{G}_{0}\right)<n$ (e.g. a pentagon). Let $p$ be a positive integer such that $n^{p} \leqq k^{2} \mu^{2 p}\left(G_{0}\right)$. Obviously, such a number exists since by our assumptions $\mu^{2}\left(G_{0}\right)=\mu\left(G_{0}\right) \cdot \mu\left(\bar{G}_{0}\right)<n$. Let $G_{k}=G_{0}^{[p]}$. It is easy to see that $\mu(G[H])=\mu(G) \cdot \mu(H)$, hence $\mu\left(G_{k}\right)=\mu^{p}\left(G_{0}\right)$. If $G$ and $H$ are self-complemented, it was shown by Sabidussi that $G[H]$ is selfcomplemented, hence $G_{k}$ is self-complemented. Consider the set $A=\left\{(g, g) \mid g \in V\left(G_{k}\right)\right\}$ as a subset of $V\left(G_{k} \times \bar{G}_{k}\right)$. Since $\left(g, g^{\prime}\right) \in E\left(G_{k}\right)$ $\Rightarrow\left(g, g^{\prime}\right) \notin E\left(\bar{G}_{k}\right)$ it follows that $A$ is an independent set of vertices in $G_{k} \times \bar{G}_{k}$. Since card $A=n^{p}$ we get

$$
\mu\left(G_{k}^{2}\right)=\mu\left(G_{k} \times \bar{G}_{k}\right) \geqq n^{p} .
$$

Ljubič [3] has shown that $\theta(G)=\lim _{n}\left(\mu\left(G^{n}\right)\right)^{1 / n}$ therefore we have in general

$$
\theta(G)=\lim _{n}\left(\mu\left(G^{2 n}\right)\right)^{1 / 2 n}=\left(\lim _{n} \mu\left(G^{2 n}\right)^{1 / n}\right)^{1 / 2}=\theta^{1 / 2}\left(G^{2}\right) .
$$

By using the lower bound for the capacity of a graph and $\left({ }^{*}\right)$ we obtain

$$
\theta\left(G_{k}\right)=\theta^{1 / 2}\left(G_{k}^{2}\right) \geqq n^{p / 2} \geqq k \mu^{p}\left(G_{0}\right)=k \mu\left(G^{[p]}\right)=k \cdot \mu\left(G_{k}\right) .
$$

\section{REFERENCES}

1. F. Harary, On the group of the composition of two graphs, Duke Math. J. 26 (1959), 29-34. MR 22 \#1523.

2. - Graph theory, Addison-Wesley, Reading, Mass., 1969.

3. A. Ljubix, A remark on a problem of C. Berge, Sibirsk. Mat. Ž. 5 (1964), 961-962. (Russian) MR 29 \#5238.

4. M. Rosenfeld, On a problem of C. E. Shannon in graph theory, Proc. Amer. Math. Soc. 18 (1967), 315-319. MR 34 \#7405.

5. C. E. Shannon, The zero error capacity of a noisy channel, IRE Trans. Information Theory IT-2 (1956), 8-19. MR 19, 623.

Louisiana State University, Baton Rouge, Louisiana 70803 